\title{
The Neutrophil Extracellular Traps: The Missed Link between Microvascular Inflammation and Diabetes?
}

\section{Berezin A*}

Internal Medicine Department, State Medical University for Zaporozhye, 26, Mayakovsky Av., Zaporozhye, 69035, Ukraine

\begin{abstract}
Microvascular inflammation is considered as a trigger for vasculopathy and early atherosclerosis development in diabetes mellitus. However, activated mononuclears/macrophages/neutrophils play a pivotal role in pathogenesis of vascular complications among individuals with diabetes mellitus. The neutrophil extracellular traps (NETs) have been known as a component of innate immune system for the last few decades. NETosis is induced by pathogens as well as other stimuli such as activated platelets, metabolic triggers, and oxidative stress components. In diabetes, neutrophils are primed to release NETs and die by NETosis. The objective of the mini review is to highlight the possible role of NETosis in early diabetes-related vasculopathy beyond cardiovascular complications.
\end{abstract}

Keywords: Diabetes mellitus; Vasculopathy; Neutrophil extracellular traps; Microvascular inflammation

\section{Introduction}

Vasculopathy plays a pivotal role in the development of cardiovascular (CV) complications in diabetes mellitus including earlyonset stage of the disease [1]. Although the molecular basis responsible for vasculopathy in diabetes mellitus has been widely investigated, the innate pathogenic mechanisms affected various phases at early stage of the disease have remained yet poorly understood [2]. Diabetes-induced vasculopathy may be attributable to hyperglycemia, lipotoxicity, neurohumoral dysregulation of vascular function, increased prolonged exposure to oxidative stress, reduced production and release of nitric oxide, low-grading microvascular inflammation, pro-thrombotic state, reduced ability to repair endothelial damage by recruitment of endothelial precursors, development of asymptomatic atherosclerosis [2-5]. Indeed hyperglycemia leads to accumulation of advanced glycation end-products (AGEs) that transduces inflammatory and proliferative response through reactive oxygen species generation, or through activation of Receptor for AGEs (RAGE)-mediated pathways [6]. The interaction of AGEs with their receptor RAGE directly elicits proliferative, inflammatory, thrombotic and fibrotic reactions in variety of cells (i.e., mononuclears/macrophages) via inducing vascular endothelial growth factor (VEGF) production and RAGE-NF- $\kappa B$ pathway activation [6,7]. Therefore, pro-inflammatory cytokines and chemokines may recruit neutrophil subsets in the vasculature wall and promote microvascular inflammation through forming neutrophil extracellular traps (NETs) [8]. However, the exact molecular mechanisms contributed in diabetes-induced vasculopathy via neutrophil extracellular traps are still not clear. The aim of the review is summary of knowledge about the role of neutrophil extracellular traps in pathophysiology of diabetes-induced vasculopathy.

\section{Definition and biological role of neutrophil extracellular traps}

The neutrophil extracellular traps have been known as a component of innate immune system for the last few decades [9]. NETs have been implicated in an increasingly large number of human diseases including infection diseases, sepsis, eclampsia, acute lung injury, thrombosis, autoimmunity, diabetes, and malignancy (Figure 1). Because NETs contain proteases, bactericidal peptides, DNA and ribonucleoprotein their main biological role is trap and kill bacteria and paracistics [10]. There is evidence that NETosis is induced by several pathogens as well as other stimuli such as activated platelets, metabolic triggers, and oxidative stress components (Figure 2).

Although the principal molecular mechanisms that involved in the activation of NETosis are not fully understood, it has been suggested that interleukin-8 (IL-8), phorbol myristate acetate (PMA), lipopolysaccharide (LPS), thromboxane $\mathrm{A}_{2}, \beta$-defensin-1, P-selectin/ $\mathrm{P}$-selectin glycoprotein ligand-1, and even simple contact of various cells with NET-releasing cells might induce NTEosis. Recent study has shown that several phospholipid mediators, i.e. peptidylarginine deiminase 4 (PAD4), are essential for NET formation [11]. PAD4 is an enzyme that converts arginine or monomethyl-arginine to citrulline in histones in a calcium dependent reaction [12]. There are four types of PAD that are distributed in nucleus and cytoplasm of granulocytes. PAD is essential for transduction of positive signals in the nucles via calcium-stimulated ionophores. However, the implication of PAD in histone modifications is discussed. One of the main mediators of histone modification and NET formation is PAF complex (Paf1C) [13]. Additionally, Paf1C has been shown to directly regulate gene transcription and RNA polymerase II elongation, coordinate several processes of adhesion and cell trafficking [14,15]. In this contest, deiminated chromatin may be internalised by host phagocytes on surface of the endothelium, which is able delimiting the NETosis via secretion of apoptotic bodies, but the innate mechanisms responsible for this response remains mysterious.

However, post-translational modifications of histones within the externalized NETs are essential component of immune complexes/ deposits and may directly damage tissue. There are evidence regarding that the nuclear DNA-binding protein HMGB1 (the high-mobility group box 1), which is released from necrotic cells/late apoptotic

*Corresponding author: Berezin A, Professor, PhD, Consultant of Therapeutic Unit, Internal Medicine Department, State Medical University for Zaporozhye, 26, Mayakovsky Av., Zaporozhye, Postcode 69035, Ukraine, Tel.: +38 0612894585 ; Fax: +38 0612894585; E-mail: dr_berezin@mail.ru

Received October 30, 2015; Accepted January 21, 2016; Published January 23 , 2016

Citation: Berezin A (2016) The Neutrophil Extracellular Traps: The Missed Link between Microvascular Inflammation and Diabetes? Metabolomics 6: 163. doi:10.4172/2153-0769.1000163

Copyright: ( 2016 Berezin A. This is an open-access article distributed under the terms of the Creative Commons Attribution License, which permits unrestricted use, distribution, and reproduction in any medium, provided the original author and source are credited. 


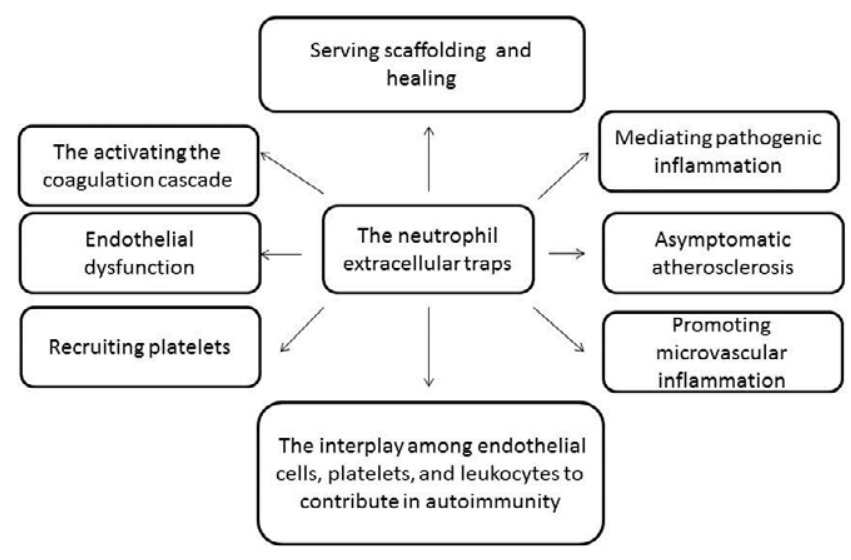

Figure 1: The implication of the neutrophil extracellular traps.

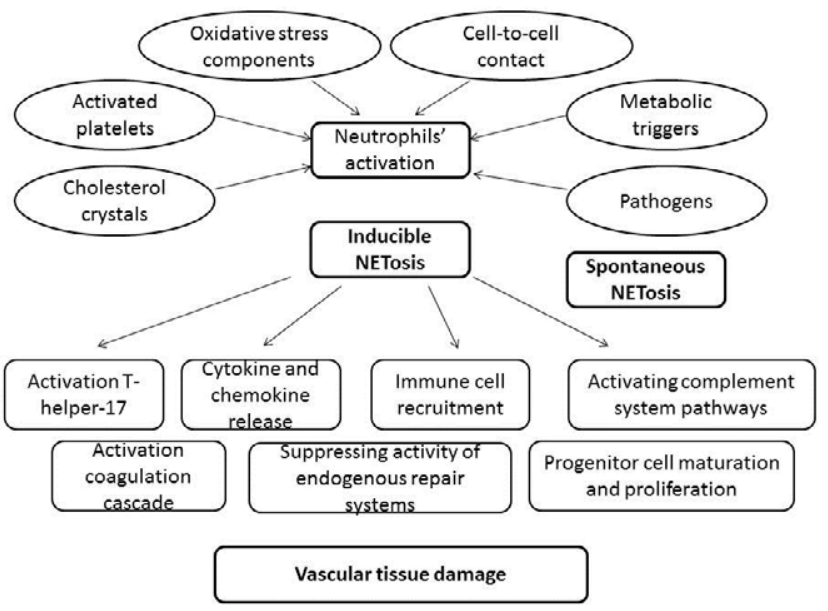

Figure 2: The molecular cascades involved in the neutrophil extracellular traps' forming.

cells and is an essential component of DNA-containing immune complexes, may stimulate cytokine production through a Tolllike receptor 9 - myeloid differentiation primary response gene- 88 pathway and induce NETosis. Indeed, HMGB1 is tightly attached to the chromatin in apoptotic cells. Moreover, HMGB1-containing nucleosomes from apoptotic cells induced secretion of interleaukin$1 \beta$, IL-6, IL-10, and tumor necrosis factor (TNF) $\alpha$ and expression of costimulatory molecules in macrophages and dendritic cells (DC), respectively [16]. Overall, HMGB1 exerts effects on NETs formation through an interaction with TLR2, TLR4 and RAGE, and this process is NADPH oxidase dependent [17]. Activated antigen-presenting cells may crucially contribute to the NETosis development and mediate the tissue damage.

Following activation by several factors mentioned above, neutrophils undergo distinct morphological alterations leading to ultimate NET formation. NETs promote the externalization of modified autoantigens, inducing synthesis of type I interferon, stimulating the formation of various inflammasomes, and activating both the classic and alternative pathways of the complement system [16]. Furthermore, NETs are considered toxic substances for the endothelium that expose immunostimulatory molecules, activate plasmacytoid dendritic cells, and may participate in target-organ damage through incompletely characterized pathways. There are at least two important targets to counter NETosis in the setting of diabetes, such as protein kinase $\mathrm{C}$ and NADPH oxidase, which modulate an abnormal response to tissue damage and immune reactions. Consequently, NETs may contribute directly to immunogenicity, endothelial cell dysfunction, vasculopathy, atherosclerotic plaque burden, and thrombosis [18]

\section{Spontaneous/inducible NETosis and diabetes-induced vasculopathy}

The ability of NETosis to induce tissue damage in infections and inflammatory disease is widely known. However, the role of spontaneous NERosis in dysmetabolic states including diabetes mellitus is not fully established. In fact, in diabetes, neutrophils are primed to release NETs and die by NETosis [19]. Neutrophils that are isolated from the blood of diabetics have been shown an increased spontaneous NETosis, but an impaired inducible exaggerated NETosis response might be a leading cause of tissue damage in diabetics with healing, such as diabetic food. Moreover, NET components (elastase, histones, NGAL, and proteinase-3) were enriched in non-healing human with diabetes [19]. In was found that the finely tuned balance of NETosis required protecting the human body from microorganisms yet avoiding self-damage seems to be lost in diabetes. Cumulatively, the ability of activated antigen-presenting cells to exhibit a spontaneous NETosis is essential for diabetes, whereas the role of inducible exaggerated NETosis remains still unclear.

There are several systemic and local stimuli affected exaggerated level of spontaneous NETosis in diabetes. Freigang et al. [20] have reported that the oxidative stress-responsive transcription factor NF-E2-related $2(\mathrm{Nrf2})$ as an essential positive regulator of inflammasome activation and IL-1-mediated vascular inflammation. Recently studies have revealed that several stimuli, i.e., local ischemia, hypoxia, cholesterol crystals accumulated in vascular wall, necrotic cells, and histones, might act both as priming signals for IL- $1 \beta$ production by and trigger for neutrophils to release NETs via represent an endogenous danger signal that activates Nrf2 and the NLRP3 inflammasome [21-23]. All these could prime macrophages for cytokine release, activating Th17 cells that amplify immune cell recruitment in atherosclerotic plaques and induce microvascular inflammation that leads to vasculopathy [24-26]. Overall, spontaneous NET-forming neutrophils may assist not only in the innate immune defense against different pathogens, but in early multicellular inflammatory reaction attenuating vascular damage and early stage of atherosclerosis.

\section{Clinical relevance of spontaneous/inducible NETosis in diabetes mellitus}

Unfortunately, there is no consensus about the clinical significance of NETosis in diabetes beyond early atherosclerosis and CV complications [27,28]. Although chronic low-grading microvascular inflammation is considered a clue in the pathogenesis of atherosclerosis, the factors that trigger and sustain the inflammation remain elusive. It has been presumed that NETs, which are functionally important players in microvascular inflammation. Indeed, NETosis contributes in tissue damage and kills endothelial/mononuclear cells and promote inflammation in atherosclerotic plaques, which may contribute to accelerated atherosclerosis. Whether classical NETosis mediates early atherosclerosis beyond plaque formation is still not clear. However, the concept of increased spontaneous NETosis that is suitable for diabetes mellitus might explain the role of low-grading microvascular inflammation as an initial stimulus for development of endothelial dysfunction beyond classical cardiovascular risk factors. 
The inflammasomes containing Nrf2 and the NLRP3 are essential regulator for inflammatory response that directly relays oxidative stress to vascular inflammation. Moreover, NETs might be target for further therapy in diabetics and that risk stratification score based on NETs' identification would be validate for prediction at early stage of diabetes irrespectively CV complications. Taking into consideration that oxidative stress and low-grading inflammation are considered the main modulator of cardiovascular complications in diabetes mellitus, spontaneous/inducible NETosis might discuss as a clue translated regulatory signal from several metabolic triggers to target cells by potentiating the inflammasome-interleukine (IL)-1 family axis and Casp1 activation. Notably, processing and secretion of bioactive IL1a and IL-1 $\beta$ be activated macrophages, are required Nrf2-regulated forming of the NLRP3 inflammasome. Interestingly, that secretion of NLRP3 inflammasome is under control of several modulators including galectine-3, which is considered as an independent predictor for CV events and disease in general population. Probably, spontaneous/inducible NETosis in diabetes mellitus might relate genetic predisposition and risk of CV complications, i.e., vasculopathy. Although the assumptions appear to be attractive, lack of clinical evidence sufficiently limits productive scientific discussion around the topic and it is required more investigations.

\section{Conclusion}

In conclusion, diabetes-related vasculopathy might be triggered by metabolic signals that presumably activating inflammasomes and spontaneous/inducible NETosis for neutrophils. Probably, NETs link the various cellular response to metabolic triggers, oxidative stress with innate microvascular inflammation mediating early cardiovascular complications in diabetes. Further investigations are required to explain the role of NETosis in pathophysiology of diabetes and possibility to use triggers of inflammasomes as targets for medical care

\section{References}

1. Schuett KA, Lehrke M, Marx N, Burgmaier M (2015) High-Risk Cardiovascular Patients: Clinical Features, Comorbidities and Interconnecting Mechanisms. Front Immunol 6: 591.

2. Bhatta A, Sangani R, Kolhe R, Toque HA, Cain M, et al. (2015) Deregulation of arginase induces bone complications in high-fat/high-sucrose diet diabetic mouse model. Mol Cell Endocrinol.

3. Vanhoutte PM, Shimokawa H, Feletou M, Tang EH (2015) Endothelial Dysfunction and Vascular Disease - A Thirthieth Anniversary Update. Acta Physiol (Oxf).

4. Leong A, Berkowitz SA, Triant VA, Porneala B, He W, et al. (2015) Hypoglycemia in diabetes mellitus as a coronary artery disease risk factor in patients at elevated vascular risk. J Clin Endocrinol Metab.

5. Lehoux S, Jones EA (2015) Shear stress, arterial identity and atherosclerosis Thromb Haemost 115.

6. Yamagishi S, Nakamura N, Suematsu M, Kaseda K, Matsui T (2015) Advanced Glycation End Products: A Molecular Target for Vascular Complications in Diabetes. Mol Med 21: S32-S40.

7. Heier M, Margeirsdottir HD, Gaarder M, Stensæth $\mathrm{KH}$, Brunborg C, et al. (2015) Soluble RAGE and atherosclerosis in youth with type 1 diabetes: a 5-year follow-up study. Cardiovasc Diabetol 14: 126.

8. Xu S, Lind L, Zhao L, Lindahl B, Venge P (2012) Plasma prolylcarboxypeptidase (angiotensinase $\mathrm{C}$ ) is increased in obesity and diabetes mellitus and related to cardiovascular dysfunction. Clin Chem 58: 1110-1115.

9. Leavy O (2015) Inflammation: NETting a one-two punch. Nat Rev Immunol 15: 526-527.

10. Kobayashi Y (2015) Neutrophil biology: an update. EXCLI J 14: 220-227.

11. Leshner M, Wang S, Lewis $C$, Zheng $H$, Chen XA, et al. (2012) PAD4 mediated histone hypercitrullination induces heterochromatin decondensation and chromatin unfolding to form neutrophil extracellular trap-like structures. Front Immunol 3: 307

12. Nakashima K, Hagiwara T, Yamada M (2002) Nuclear localization of peptidylarginine deiminase $\mathrm{V}$ and histone deimination in granulocytes. J Bio Chem 277: 49562-49568.

13. Yang Y, Li W, Hoque M, Hou L, Shen S, et al. (2016) PAF Complex Plays Novel Subunit-Specific Roles in Alternative Cleavage and Polyadenylation. PLoS Genet 12: e1005794.

14. Zhu B, Mandal SS, Pham AD, Zheng Y, Erdjument-Bromage $\mathrm{H}$, et al. (2005) The human PAF complex coordinates transcription with events downstream of RNA synthesis. Genes Dev 19: 1668-16673.

15. Chen FX, Woodfin AR, Gardini A, Rickels RA, Marshall SA, et al. (2015) PAF1, a Molecular Regulator of Promoter-Proximal Pausing by RNA Polymerase II. Cell 162: 1003-1015.

16. Wang H, Wang C, Zhao MH, Chen M (2015) Neutrophil extracellular traps can activate alternative complement pathways. Clin Exp Immunol 181: 518-527.

17. Ma YH, Ma TT, Wang C, Wang H, Chang DY, et al. (2016) High-mobility group box 1 potentiates antineutrophil cytoplasmic antibody-inducing neutrophil extracellular traps formation. Arthritis Res Ther 18: 2

18. Grayson PC, Kaplan MJ (2015) At the Bench: Neutrophil extracellular traps (NETs) highlight novel aspects of innate immune system involvement in autoimmune diseases. J Leukoc Biol.

19. Fadini GP, Menegazzo L, Rigato M, Scattolini V, Poncina N, et al. (2016) NETosis delays diabetic wound healing in mice and humans. Diabetes.

20. Freigang S, Ampenberger F, Spohn G, Heer S, Shamshiev AT, et al. (2011) Nrf2 is essential for cholesterol crystal-induced inflammasome activation and exacerbation of atherosclerosis. Eur J Immunol 41: 2040-2051.

21. Warnatsch A, loannou M, Wang Q, Papayannopoulos V (2015) Inflammation. Neutrophil extracellular traps license macrophages for cytokine production in atherosclerosis. Science 349: 316-320.

22. Nahrendorf M, Swirski FK (2015) Immunology. Neutrophil-macrophage communication in inflammation and atherosclerosis. Science 349: 237-238.

23. Allam R, Darisipudi MN, Tschopp J, Anders HJ (2013) Histones trigger sterile inflammation by activating the NLRP3 inflammasome. Eur J Immunol 43: 3336 3342

24. Rajamäki K, Lappalainen J, Oörni K, Välimäki E, Matikainen S, et al. (2010) Cholesterol crystals activate the NLRP3 inflammasome in human macrophages: a novel link between cholesterol metabolism and inflammation. PLoS One 5 : e11765.

25. Rajamäki K, Nordström T, Nurmi K, Åkerman KE, Kovanen PT, et al. (2013) Extracellular acidosis is a novel danger signal alerting innate immunity via the NLRP3 inflammasome. J Biol Chem 288: 13410-13419

26. Freigang S, Ampenberger F, Weiss A, Kanneganti TD, Iwakura Y, et al. (2013) Fatty acid-induced mitochondrial uncoupling elicits inflammasome-independent IL-1 $\alpha$ and sterile vascular inflammation in atherosclerosis. Nat Immunol 14 1045-1053.

27. Mangold A, Alias S, Scherz T, Hofbauer T, Jakowitsch J, et al. (2015) Coronary neutrophil extracellular trap burden and deoxyribonuclease activity in STelevation acute coronary syndrome are predictors of ST-segment resolution and infarct size. Circ Res 116: 1182-1192.

28. Michel JB, Ho-Tin-Noé B (2015) Thrombi and neutrophils. Circ Res 116: 1107 1108. 